\title{
10 - year experience in the application of biological therapy to patients with juvenile idiopathic arthritis
}

\section{Zaure Mukusheva', Maikesh Asylbekova', \\ ${ }^{1}$ Department of rheumatology of the Corporate Fund "University Medical Center» National Research Center for Maternal and Child Health, Astana, Kazakhstan.}

This work is licensed under a Creative Commons Attribution 4.0 International License

J CLIN MED KAZ 2017; 3(45 SUPPL 3):159-162 Corresponding author: Мукушева Зауре Серикпаевна, Отделение ревматологии Филиала корпоративного фонда "University Medical Center" Национального научного центра материнства и детства. Адрес:010000, пр. Туран 32, г. Астана, Казахстан, тел. 701439,

e-mail: mukusheva@inbox.ru

\section{ABSTRACT}

The article presents the experience of using biological agents for the treatment of children with juvenile idiopathic arthritis in the department of rheumatology of the Corporate Fund "University Medical Center" of the National Research Center for Maternal and Child Health.

Key words: children, juvenile idiopathic arthritis, biological preparations, anti -TNFdrugs, anti-interleukin- 6 drugs.

ТҰЖЫРЫМДАМА

ЮВЕНИЛЬДІ ИДИОПАТИКАЛЫҚ АРТРИТПЕН АУЫРАТЫН

НАУҚАСТАРҒА БИОЛОГИЯЛЫК ТЕРАПИЯНЫ ҚОЛДАНУ БОЙЫНША 10 жЫЛДЫҚ ЖҰМЫС ТӘЖІРИБЕСІ

Мукушева 3.С.1, Асылбекова М.К.1, Альбекова Ж.О.1, Садибекова Л.Д.1

'«University Medical Center» Корпоративтік қорының Ана мен бала ұлттық ғылыми орталығы, ревматология бөлімі, Астана, Қазакстан

Мақалада Ана мен бала Ұлттықғылыми орталығының «Университет медициналық орталығы» Корпоративтік қорының фрилиалының ревматология бөлімінде ювенильді идиопатикалық артритпен емдеуге арналған биологиялық агенттерді қолдану тәжірибесі берілген.

Түйінді сөздер: балалар, ювенильді идиопатикалық артрит, биологиялық препараттар, анти-интерлейкин-6 препараттары.

\section{0 - ЛЕТНИЙ ОПЫТ ПРИМЕНЕНИЯ БИОЛОГИЧЕСКОЙ ТЕРАПИИ} ПАЦИЕНТАМ С ЮВЕНИЛЬНЫМ ИДИОПАТИЧЕСКИМ АРТРИТОМ Мукушева 3.С.1, Асылбекова М.К.1, Альбекова Ж.О.1, Садибекова Л.Д.1

'Национальный научный центр материнства и детства корпоративного фонда «UniversityMedicalCenter», Отделение ревматологии, Астана, Казахстан

В статье представлен опыт применения биологических препаратов для лечения детей с ювенильным идиопатическим артритом в отделении ревматологии Филиала корпоративного фонда "University Medical Center" Национального научного центра материнства и детства.

Ключевые слова: дети, ювенильный идиопатический артрит, биологические препараты, анти ФНО -препараты, анти- интерлейкин-6 препараты.

\section{Введение}

Ювенильный идиопатический артрит (ЮИА)заболевание неизвестного происхождения с воспалением в одном или нескольких суставах, у детей в возрасте до 16 лет, продолжительностью не менее 6 недель, при исключении других возможных причин повреждения суставов - по данным Международной лиги ассоциаций ревматических заболеваний(ILAR) [1].

Первичная заболеваемость ЮИА в Казахстане в 2016 году - 14,48 на 100000 детского населения. В РК наблюдается тенденция к увеличению уровня распространенности на $49,5 \%$ и первичной заболеваемости на $65,3 \%$ в 2016 году в сравнении с 2014 годом (рисунок 1) [2].

Распространенность ЮИА составила 32,82 на 100000 детского населения. По данным официальной статистики РК на 2016 год зарегистрировано 1739 детей с ЮИА, в том числе 1305 детей в возрасте до 14 лет и 434 - в возрасте 1517 лет, из которых 51,3\% девочки[2].

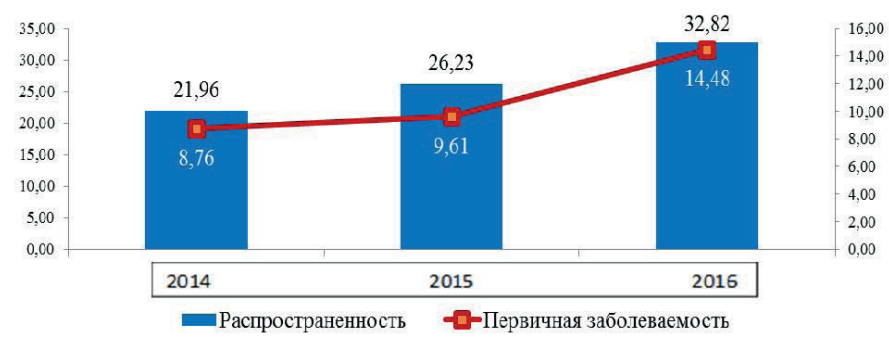

гисунок I - динамика уровня распространенности и первичной заболеваемости ЮИА на 100000 детского населения в РК 
B соответствии с критериями ILAR [1]ЮИА делится на семь подтипов: системный артрит (M08.2), олигоартрит (М08.4), РФ отрицательный полиартрит (М08.3), РФ положительный полиартрит (М08.8), энтезитассоциированные артриты (М08.1), псориатический артрит (М08.8) и недифференцированные артриты (М08.9). Данная классификация обусловлена этиопатогенезом и существующими различиями клинического течения заболевания.

Структура ЮИА у пациентов, получавших лечение в отделении ревматологии КФ «UМС» ННЦМД в 2016 году, представлена на рисунке 2 [20,21].

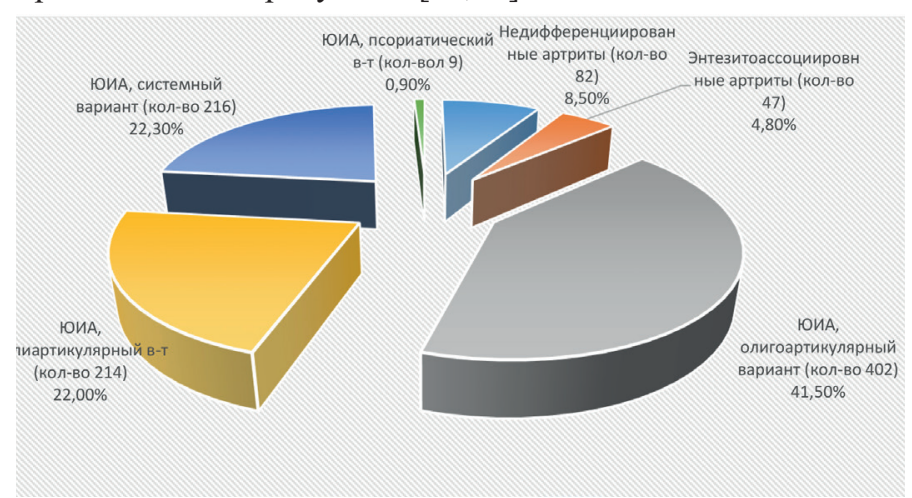

Рисунок 2 - Структура ЮИА отделения ревматологии ННЦМД за 2016 год

Исследования последних 10 лет показали, что от 40 до 60 \% пациентов, получавших длительно нестероидные противовоспалительные препараты, базисную терапию (метотрексат, сульфасалазин) достигают ремиссию заболевания [3-7]. Тем не менее, более $30 \%$ детей остаются с активным ЮИА, что приводит к серьезным осложнениям, таким как нарушение физического развития, задержку роста, остеопороз, синдром активации макрофагов, иридоциклит и нарушения зрения [3-7]. В терапии таких пациентов оправдано применение генно-инженерных биологических препаратов (ГИБП), позволяющее достичь ремиссии или неактивной фазы болезни, что значительно снижает инвалидизацию детей с ЮИА [8-18].

На рисунке 3 представлено соотношение терапии ГИБП и базисной терапии ЮИА в Казахстане в 2012 году и в 2016 году[20-22].
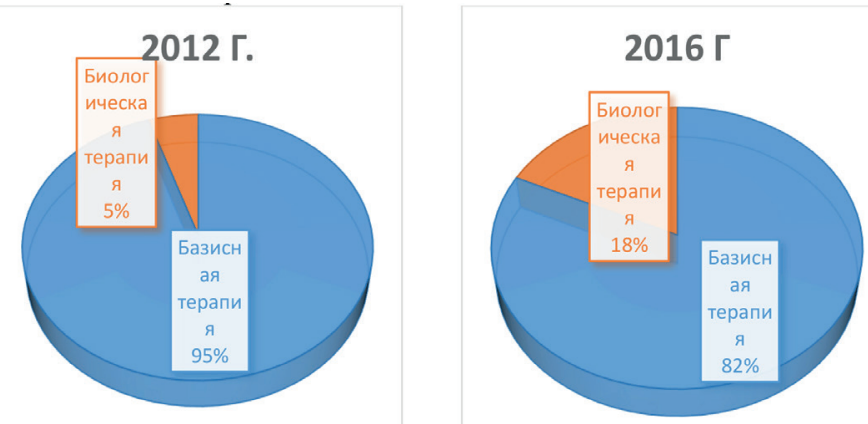

Рисунок 3 - Терапия ЮИА в Казахстане в 2012 и 2016 годах

Для постановки диагноза и определения стратегии терапии ЮИА за основу были взяты критерии, рекомендованные Американской коллегией ревматологии (ACR) 2011 и 2013 годов[4,18]. На их основе был разработан клинический протокол ЮИА, который был утвержден на заседании Экспертной Комиссии по вопросам развития здравоохранения Министерства Здравоохранения РК от 05 мая 2014 года[19]. Согласно этому протоколу, основу терапии ЮИА составляет назначение иммуносупрессивных препаратов, среди которых препаратом первого ряда является Метотрексат. При неэффективности терапии Метотрексатом в течение 3-6 месяцев, назначаются ГИБП. Соответственно вышеуказанному клиническому протоколу в РК могут использоваться 3 ГИБП: Этанерцепт, Адалимумаб, Тоцилизумаб в зависимости от варианта ЮИА; данные препараты, включены в Республиканский лекарственный формуляр, Казахстанский Национальный формуляр.

Опыт использования препарата Инфликсимаб у 57 пациентов с ЮИА продемонстрировал, что 5 пациентов не имели терапевтического ответа на терапию Инфликсимабом - это были пациенты с системным вариантом ЮИА, которым показаны блокаторы интерлейкинов, не имевшиеся в наличии на тот период времени. 1 пациент реализовал туберкулезную инфекцию[20-22]. Также можно отметить частые аллергические реакции на инфузию Инфликсимаба, связанные с тем, что он содержит химерные мышиночеловеческие антитела (рисунок 4). Наряду с этим следует отметить высокую эффективность препарата у пациентов с полиартикулярным и энтезит-асссоциированным вариантами ЮИА.

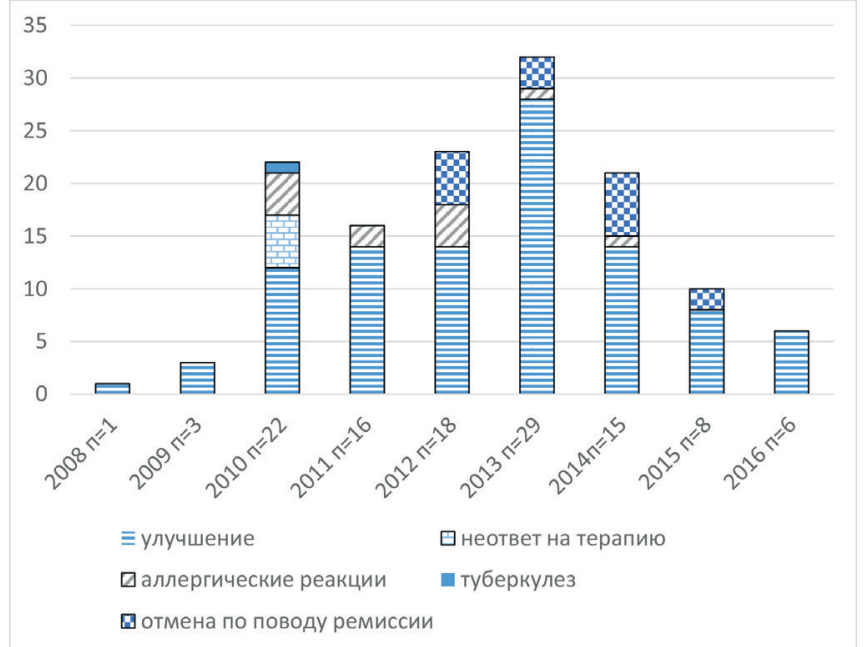

Рисунок 4 - Терапия Инфликсимабом

В последующем, в связи с отсутствием четких показании к применению Инфликсимаба в терапии ЮИА, нуждающиеся в продолжении биологическойтерапии переведены на другие анти-ФНО препараты (Этанерцепт, Адалимумаб).

В период с 2011-2013 годы терапия Адалимубамом применялась у 3 пациентов с персистирующей активностью увеита. По данным 2016 года, из 56 пациентов на терапии Адалимумабом 48 пациентов $(85,7 \%)$ имели поражение глаз по типу увеита [20-22]. Следует отметить отсутствие серьезных нежелательных явленийв момент терапии данным препаратом, и высокую клиническую эффективность в виде быстрого достижения ремиссии (рисунок 5). Препарат считается перспективным в лечении ЮИА ассоциированных увеитов с хроническим непрерывно-рецидивирующим и резистентным к базисной терапии течением у детей. При этом даже с первого года терапии Адалимумабом имеются пациенты резистентные к данной терапии и нуждающиеся в применении второй линии терапии блокаторами костимуляции Т-лимфоцитов - Абатацептом (Оренсия). 


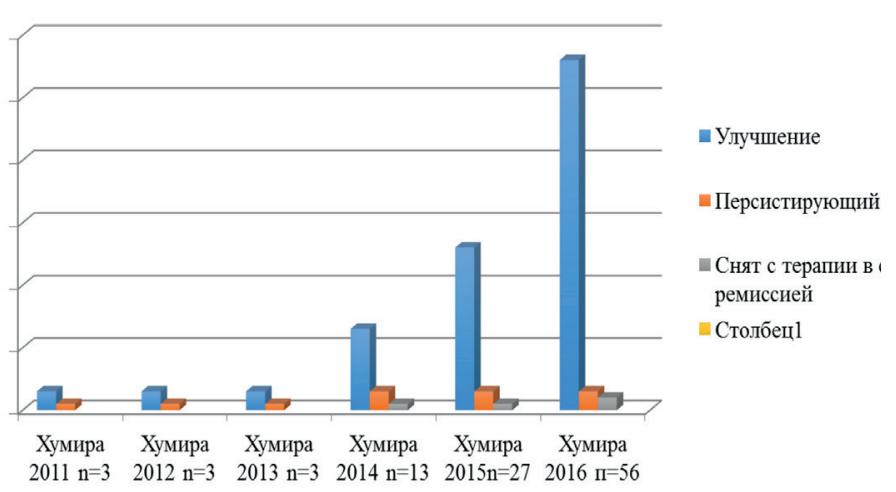

Рисунок 5 - Терапия Адалимумабом

Терапия Этанерцептом была проведена 52 пациентам, из них 11 детей с олигоартикулярным распространяющимся вариантом, 33 ребенка с полиартикулярным вариантом и 8 детей с энтезит-ассоциированным вариантом [20-22]. На фоне терапии Этанерцептом серьёзных нежелательных явлении не зарегистрировано. Необходимо отметить, что 2 пациентов с реализацией увеита, были переведены на терапию Адалимумабом. У 1 пациента отмечалась аллергическая реакция, потребовавшая отмену препарата (рисунок 6). У большинства пациентов отмечена высокая эффективность. При комплексной оценке критерииACRpedi -30 был достигнут у 100\%, ACRpedi -50 у 66,6\%, ACRpedi -70 y $47,6 \%$, ACRpedi $-80-28,5 \%$.

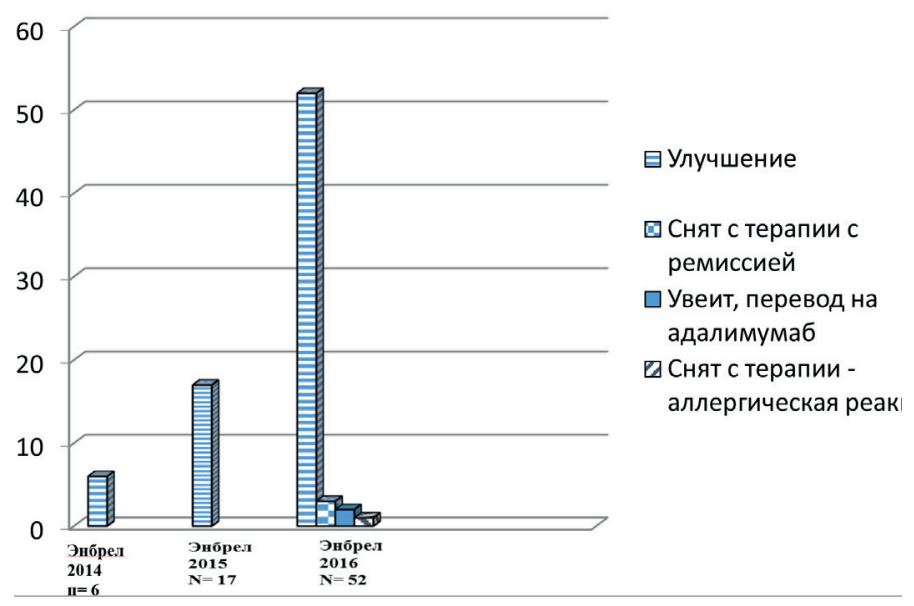

Рисунок 6 - Терапия Этанерцептом

Терапия Тоцилизумабом проводилась 81 пациенту, из них с полиартикулряным вариантом составили 12 пациентов, системным вариантом 69 пациента. Необходимо отметить, что 17 пациентов (24,6\%) с системным артритом на монотерапии - это пациенты с ранним дебютом ЮИА. У пациентов с системным ЮИА после первой инфузии Тоцилизумаба лихорадка купировалась у 96\%, кожный синдром прекратился через 12 недель у 85\%, через 6 месяцев отмечалось купирование суставного синдрома. Из серьезных нежелательных явлении наблюдались аллергические реакции у 6 пациентов, лейкопения у 2 пациентов, синдром активации макрофагов со смертельным исходом 1, токсический гепатит у 4 (рисунок 7). Также следует отметить, что у 8 пациентов с сохраняющейся персистирующей активностью заболевания, на фоне терапии Тоцилизумабом отмечалисьвоспалительный характер крови, системные проявления и полиартрит. Наряду с этим следует отметить высокую эффективность терапии по критериям ACRpedi через 6 мес 30/50/70/90\% улучшения было зарегистрировано у 100/75/65/50\% больных соответственно. В связи с достижением клинической ремиссии, снято с терапии 23 пациента с системным вариантом ЮИА, в дальнейшем у 3 из них развилсярецидив заболевания с возвратом к терапии [20-22].

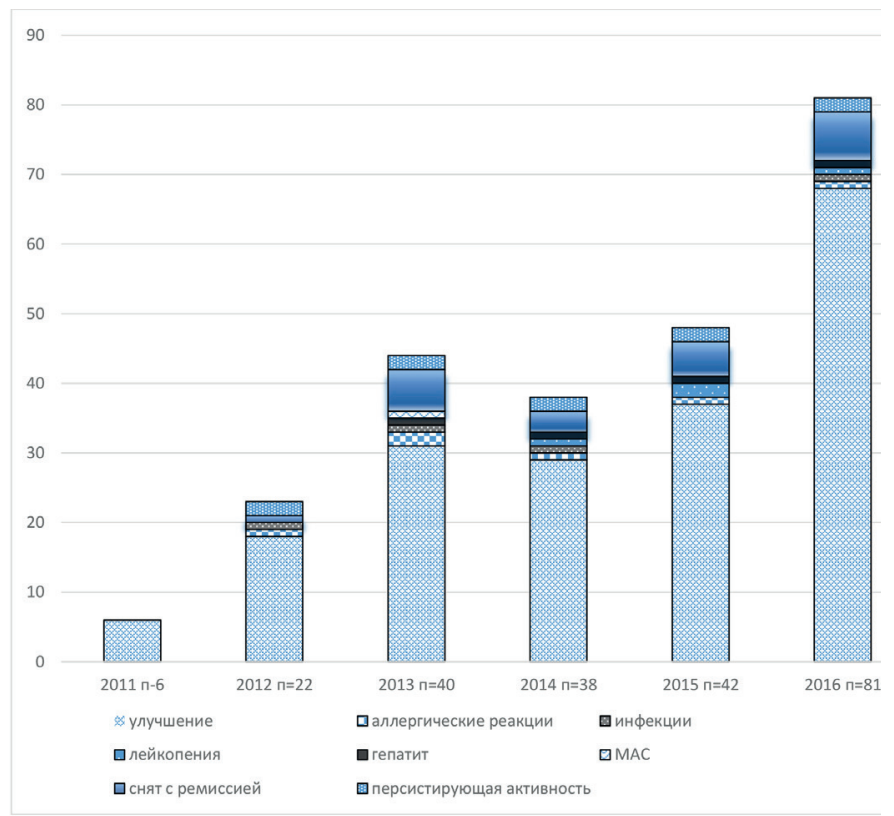

Рисунок 7 - Терапия Тоцилизумабом

\section{Выводы}

За 10 лет работы отделения ревматологии КФ «UMC» ННЦМД терапия биологическими препаратами была проведена195 пациентамс ЮИА[20,21].Лечение ГИБП открыло реальные возможности эффективной лечебной помощи пациентам с ЮИА, включая перспективу существенного снижения риска инвалидизации, отмену в терапии глюкокортикоидов, увеличение случаев ремиссии с полной отменой медикаментов.

Учитывая опыт ведения больных с системным вариантом ЮИА, можно заключить что наряду с достигнутыми успехами в терапии, у $10 \%$ пациентов существует проблема резистентностик терапии Тоцилизумабом, которая требует назначения блокаторов интерлейкина-1, не зарегистрированных в РК на сегодняшний день. Это препараты: Анкинра (Кинерет), Канакинумаб (Иларис). А также блокатор ко-стимуляции Т-лимфоцитов - Абатацепт (Оренсия) для пациентов с увеитом, резистентных к терапии Адалимумабом. 


\section{Литература:}

1. Petty R. E., Southwood T. R., Manners P., et al. International League of Associations for Rheumatology classification of juvenile idiopathic arthritis: second revision, Edmonton, 2001. JRheumatol 2004; 31:390-2.

2. Godovajaforma №12 "Otchetochislezabolevanij, zaregistrirovannyhubol'nyh, prozhivajushhihvrajoneobsluzhivanijamedicin skojorganizaciiikontingentahbol'nyh, sostojashhihpoddispansernymnabljudeniem, dannyeMZRK 2016. (Annual report No. 12 "Report on the number of diseases registered in patients living in the service area of the medical organization and the contingent of patients under dispensary supervision, data from the Ministry of Health of the Republic of Kazakhstan 2016) [in Russian].

3. Andersson Gare B. Juvenile arthritis: who gets it, where and when? A review of current data on incidence and prevalence. ClinExpRheumatol. 1999; 17:367-74.

4. Beukelman T., Patkar N. M., Saag K. G., et al. 2011 American College of Rheumatology Recommendations for the Treatment of Juvenile Idiopathic Arthritis: Initiation and Safety Monitoring of Therapeutic Agents for the Treatment of Arthritis and Systemic Features. Arthritis Care \& Research. 2011; 63:465-482.

5. Selvaag A. M., Flato B., Dale K., Lien G., Vinje O., Smerdel-Ramoya A., et al. Radiographic and clinical outcome in early juvenile rheumatoid arthritis and juvenile spondyloarthropathy: a 3-year prospective study. J Rheumatol. 2006; 33:1382-91.

6. Packham J. C., Hall M. A. Long-term follow-up of 246 adults with juvenile idiopathic arthritis: functional outcome. Rheumatology (Oxford).2002; 41:1428-35.

7. Raveli A., Martini A. Juvenile idiopatic arthritis. Lancet. 2007; 369(9563):767-778.

8. Lovell D. J., Reiff A., Ilowite N. T., Wallace C. A., Chon Y., Lin S. L., et al, for the Pediatric Rheumatology Collaborative Study Group. Safety and efficacy of up to eight years of continuous etanercept therapy in patients with juvenile rheumatoid arthritis. Arthritis Rheum. 2008; 58:1496-504.

9. Lovell D. J., Ruperto N., Goodman S., Reiff A., Jung L., Jarosova K., et al. Adalimumab with or without methotrexate in juvenile rheumatoid arthritis. N Engl J Med. 2008; 359:810-20.

10. Pascual V., Allantaz F., Arce E., Punaro M., Banchereau J. Role of interleukin-1 (IL-1) in the pathogenesis of systemic onset juvenile idiopathic arthritis and clinical response to IL-1 blockade. J Exp Med. 2005; 201:1479-86.

11. Ruperto N., Lovell D. J., Quartier P., Paz E., Rubio-Perez N., Silva C. A., et al. Abatacept in children with juvenile idiopathic arthritis: a randomised, double-blind, placebo-controlled withdrawal trial. Lancet. 2008; 372:383-91.

12. Lovell DJ, Giannini EH, Reif A., et al, for Pediatric Rheumatology Collaboratve Study Group. Etanercept in children with polyarticular juvenile rheumatoid arthritis. N Engl J Med. 2000; 324: 763-9.

13. Horneff G., De Bock F., Foeldvari I., Girschick H. J., Michels H., Moebius D., et al. Safety and efficacy of combination of etanercept and methotrexate compared to treatment with etanercept only in patients with juvenile idiopathic arthritis (JIA): preliminary data from the German JIA Registry. Ann Rheum Dis. 2009; 68:519-25.

14. Lovell D., Ruperto N., Goodman S, et al. Adalimumab with or without methotrexate in juvenile rheumatoid arthritis. $N$ Engl $J$ Med. 2008; 359(8):810-820

15. Herlins T. Tocilizumab:the evidence for its place in the treatment of juvenile idiopathic arthritis. Core Evidence. 2009 ; 4:181-189.

16. Ruperto N, Lovell DJ, Quartier P, et al. Abatacept in children with JIA: A randomised, double-blind, placebo-controlled withdrawal trial. Lancet. 2008; 372(9636): 383-391.

17. Biester , Deuter C, Michels H, Haefner R, Kuemmerle J. Adalimumab in the therapy of uveitis in childhood. Br J Ophthalmol. 2007; 91(3): 319-324.

18. Sarah Ringold, Pamela F. Weiss, Timothy Beukelman et al. 2013 Update of the 2011 American College of Rheumatology Recommendations for the Treatment of Juvenile Idiopathic Arthritis Recommendations for the Medical Therapy of Children With Systemic Juvenile Idiopathic Arthritis and Tuberculosis Screening AmongChildren Receiving Biologic Medications. Arthritis\&Rheumatism. 2013;65(10):2499-2512.

19. Sbornikklinicheskihprotokolovpoprofiljupediatrija (Collection of clinical protocols on the profile of pediatrics) [in Russian]. 1th ed. 2014. 161-188 p.

20. Otchetnaja forma № 14 «Svedenija o dejatel'nostistacionara», za 2008-2016gody, dannyeotdelastatistiki KF «UMC» NNCMD (Report form No. 14 "Information on the activities of the hospital", for 2008-2016years, data of the statistics department of the CF "UMC" NNCMD) [in Rusiian].

21. Otchetnaja forma № № 30 «Svedenijaobuchrezhdeniizdravoohranenija», za 2008-2016gody, dannyeotdelastatistiki KF «UMC» NNCMD (Report form No. 30 "Information on the establishment of health care", for 2008-2016, data of the Statistics Department of the "UMC" CFMC.) [in Russian].

22. Otchetpo 007 programme «Perspektivnyefeto-infantnyetehnologiisnizhenijareproduktivnyhidetskihpoter'» AO «Nacional'nyj nauchnyjcentrmaterinstvaidetstva» ponapravleniju «Optimizacijadiagnostikiilechenijajuvenil'nyhhronicheskihartritov» 20112013gg. (Report on 007 program "Perspective feto-infant technologies for reducing reproductive and childhood losses" JSC "National Research Center for Maternity and Childhood" in the direction "Optimization of diagnosis and treatment of juvenile chronic arthritis" 2011-2013gg) [in Russian]. 